\title{
Participación de la Comunidad Educativa Escolar, factor de éxito, en el diseño y ejecución del Plan de Mejora
}

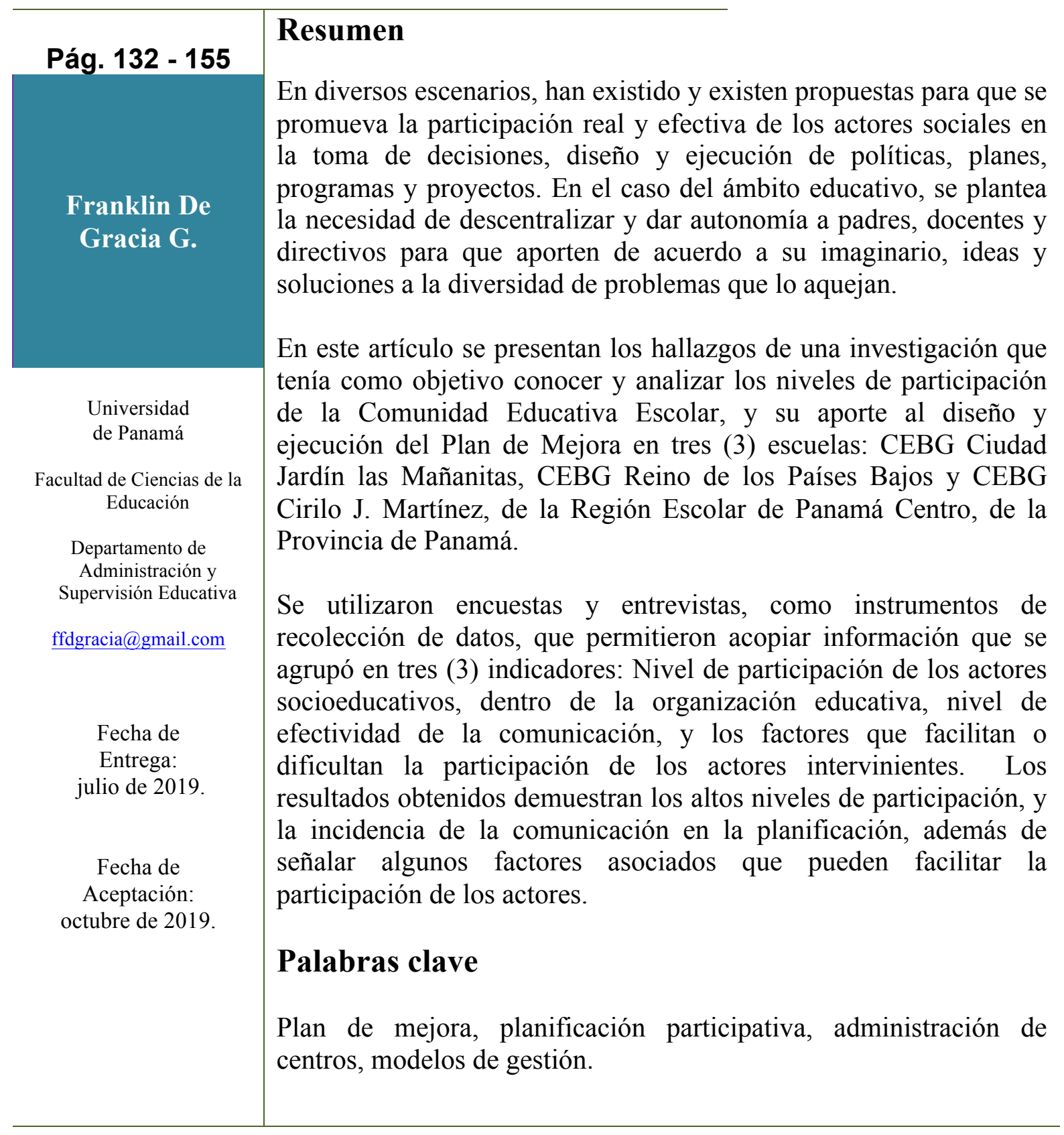

http://revistas.up.ac.pa 


\title{
Participation of the School Educational Community: a success
} factor in the design and execution of the Improvement Plan

\begin{abstract}
In various scenarios, there have been presented proposals to promote the real and effective participation of social actors in decision making, design and implementation of policies, plans, programs and projects. In the case of education, it is proposed the need to decentralize and empower parents, teachers and managers, so they could contribute with ideas to solve the diversity of problems that afflict them.
\end{abstract}

This article presents the findings of a research aiming at knowing and analyzing the participation levels of the School Educational Community, and its contribution to the design and execution of the Improvement Plan in three schools: Ciudad Jardin Las Mañanitas CEBG (General Basic School Center), Reino de los Paises Bajos CEBG and Cirilo J. Martinez CEBG, Panama Metropolitan School Region, Province of Panama.

Surveys and interviews were used as data collection instruments, which allowed to collect information from three indicators: Participation level of socio-educational actors within the educational organization, effectiveness level of communication, and factors that facilitate or hinder the participation of actors involved. The results obtained prove the high levels of participation, and the incidence of communication in planning, in addition to figuring out some associated factors that can facilitate the participation of actors.

\section{Keywords}

Improvement Plan, participatory planning, school management, management models.

REVISTA ANUAL ACCIÓN Y REFLEXIÓN EDUCATIVA, N 45

enero, 2020

ISSN L 2664-3775 


\section{INTRODUCCIÓN}

El presente escrito pretende responder y aportar información a la comunidad educativa en relación con la siguiente pregunta: ¿Cuáles son los niveles de participación de la Comunidad Educativa Escolar, en el diseño y ejecución del Plan de Mejora Escolar? La pregunta de la investigación planteada intenta encontrar la relación existente entre las siguientes variables: 1) la participación de los miembros de la comunidad educativa alrededor del proceso de planificación, 2) su aporte en el diseño y ejecución del Plan de Mejora Escolar.

Además de la pregunta central del problema era obligante realizar algunas más:

¿Cómo participan los padres de familia en la organización educativa de las escuelas de la Región Escolar de Panamá Centro?

- ¿Cómo se relacionan los docentes con los padres de familia y otros miembros de la comunidad educativa escolar y cómo aportan los para docentes a satisfacer las inquietudes de estos?

- ¿Qué motivaciones tienen los padres de familia para participar en la organización integral del centro?

- ¿Qué factores dificultan esta participación?

- ¿Qué factores facilitan la participación?

- ¿Qué demandas presentan los padres de familia y otros miembros de la comunidad educativa escolar, frente a la realidad educativa del centro?

Los antecedentes y el estudio sobre la literatura en torno a la planificación y la participación colectiva sustentaron en gran medida al planteamiento del problema de la investigación.

REVISTA ANUAL ACCIÓN Y REFLEXIÓN EDUCATIVA, N 45

enero, 2020

ISSN L 2664-3775 
A finales de la administración gubernamental 2009-2014, el Ministerio de Educación de Panamá se abocó a la creación del Sistema de Evaluación de Centros Educativos (SECE), lo que se tradujo en el diseño de un modelo de evaluación de centros educativos, que constó de cuatro (4) fases 1. Auto evaluación del centro educativo. 2. Elaboración de un Plan de Mejora, 3. Seguimiento implementación y evaluación del Plan de Mejoras. 4. Evaluación y certificación de calidad institucional.

Luego de culminar la primera y segunda fase del proceso de evaluación, surgieron algunas críticas al modelo y a la implementación de éste, por algunos miembros de la comunidad educativa nacional, por lo que se creyó conveniente realizar un estudio que ofreciera respuestas a la luz de una estructura sistematizada y científica.

En base a estos antecedentes este artículo esboza los resultados de una investigación que se propuso indagar la temática a través de la información existente, encuesta y entrevista a los miembros de la comunidad educativa de las escuelas: CEBG Ciudad Jardín las Mañanitas, CEBG Reino de los Países Bajos y CEBG Cirilo J. Martínez, de la Región Educativa de Panamá Centro, a fin de encontrar respuestas y sistematizar el conocimiento resultante.

No obstante, es importante dejar constancia, en cuanto a la planificación normativa, prospectiva o estratégica que existen en nuestro medio referentes de investigaciones revistas, libros y otras publicaciones, que dedican espacio a la temática y al análisis oportuno de las fortalezas y debilidades de cada modelo de planificación.

\section{MARCO TEÓRICO ORIENTADOR}

Los discursos teóricos que fundamentan esta investigación explicitan teorías sobre los Planes de Mejoras, que las escuelas de la Región Escolar de Panamá Centro proponen, y si estos son más efectivos si se hacen de manera colaborativa, es decir si se integran todos los miembros de la

REVISTA ANUAL ACCIÓN Y REFLEXIÓN EDUCATIVA, N 45

enero, 2020

ISSN L 2664-3775 
Comunidad Educativa Escolar. Esto, lo garantiza el trabajo en equipo, dando como resultado un plan que se convierta en la "hoja de ruta", para enfrentar el futuro y sus problemáticas; el cual facilita materializar oportunidades para ser eficiente y eficaz, por medio de la definición de metas y acciones concretas, que movilizan todos los aspectos de la gestión escolar hacia el logro de los resultados esperados.

Al respecto Antúnez (2012), señala que:

Todos los miembros de la comunidad educativa, en función de sus capacidades y competencias, pueden intervenir en la gestión del centro. En el caso de los profesores y profesoras, la participación es intrínseca a su trabajo profesional. A los demás miembros de la comunidad educativa les asisten sus derechos democráticos (p.44).

Es evidente que "la participación hace realidades tangibles e intangibles dentro de las organizaciones, especificamente las educativas"; tal como lo afirma Santos (2007), en (Harf \& Azzerboni, 2009, p.45). Los integrantes de la comunidad educativa tienen entre sus manos las claves de la comprensión. Ellos saben como participantes activos, qué es lo que está sucediendo en el seno de la organización escolar de los CEBG. Pero, lo más importante no es sólo que pueden saber lo que sucede, es que poseen las capacidades y competencias en la gestión para transformarlos en modelos óptimos. Las concepciones esbozadas permitieron hacer explicaciones de acuerdo con los alcances y magnitudes, que consintieron en amplificar, grosso modo, las teorizaciones siguientes: Planes de mejora como alternativa de gestión participativa; plan de mejora al contexto de los centros educativos panameños; evaluación institucional (Participación de los actores socioeducativos); Los procesos del SECE (autoevaluación, plan de mejoras); Aplicación del plan de mejora.

REVISTA ANUAL ACCIÓN Y REFLEXIÓN EDUCATIVA, N 45

enero, 2020

ISSN L 2664-3775 


\section{- Planes de mejora como alternativa de gestión participativa}

Existen antecedentes relacionados con la gestión participativa y su relación con la planificación dentro de los procesos administrativos. Salinas (2003) realizó una investigación en el Instituto Tecnológico de Monterrey. Dicha investigación se desarrolló en tres etapas: Primera: dimensión empírica. Abordar la realidad de la práctica actual de la planeación institucional de la escuela en estudio. Segunda: dimensión teórica. Construir un marco teórico para comprender la realidad empírica y la Tercera: dimensión diagnóstica.

Esta investigación buscaba confrontar la realidad empírica-teórica, para establecer la distancia entre ambas realidades y visualizar posibles cambios tendientes a mejorar la práctica actual de la planeación institucional de la escuela en estudio; para posteriormente elaborar un diagnóstico general, como actividad interpretativa y dar significado a la situación estudiada, facilitando la delimitación y precisión de posibles mejoras a la realidad de la práctica de la planeación; y por último valorar las posibles mejoras de la situación dictaminada en el diagnóstico.

Otro antecedente tomado en cuenta fue el estudio de Alarcón, (2008) que fue realizado en la Universidad Nacional Experimental de Guayana, República Bolivariana de Venezuela, cuyo objetivo planteado era "diseñar un modelo de planificación estratégica que permita articular el trabajo de las diferentes unidades de apoyo y del cuerpo docente, como centro de fortalecimiento de las potencialidades humanas y eje del desarrollo local en un entorno cambiante y de escasos recursos" (p.6). Los resultados conclusivos en tal estudio, especifican la necesidad inminente de aplicación de una planificación estratégica, de modo que el director del plantel gestione con mayor autonomía los recursos de que dispone en pro de alcanzar la misión y la visión organizacional, empleando para ello el conjunto de estrategias que resultan del análisis de la organización.

REVISTA ANUAL ACCIÓN Y REFLEXIÓN EDUCATIVA, N 45

enero, 2020

ISSN L 2664-3775 
Tomada esta experiencia foránea y de manera análoga con las prácticas de aplicación de la planificación estratégica que se desarrolló en Panamá, y en donde el Ministerio de Educación (MEDUCA) establece en sus políticas educativas, la regionalización educativa como estrategia de planificación y administración del servicio educativo (Ley 34, modifica la Ley 47 de 24 de septiembre de 1946). Ante esta realidad el Artículo 38 de la precitada Ley Orgánica, expresa que la República de Panamá se divide en regiones escolares, determinadas de acuerdo con el número de centros educativos y de educadores, así como por las facilidades de comunicación.

Con posterioridad se reglamentó esta subdivisión, misma que se expresa cómo circunscripción territorial de las Regiones Escolares: ... "La Región Escolar de Panamá Centro comprende la circunscripción territorial del distrito de Panamá, con excepción de los corregimientos de Las Cumbres y Chilibre.”... (Decreto Ejecutivo 446 de 21 de noviembre de 2006, Art. 2).

En el ámbito organizacional, algunos teóricos de la gestión educativa argumentan que para que exista una razonable misión eficiente y responsable en los centros escolares, se hace necesaria la práctica de procesos gerenciales, es decir, fundamentados en un modelo de gestión definido, que conlleve el mejoramiento activo y dinámico desde las autoridades escolares veladoras del funcionamiento material (equipamientos e infraestructuras edificadas y suministros de apoyo) e inmaterial (docentes motivados por sus saberes y quehaceres académicos, incentivos y reconocimientos por sus labores docentes, fidelizaciones para con los centros escolares, compromisos por las enseñanzas a los estudiantes y viceversa las fidelidades y responsabilidades de los estudiantes), tales encargos, vislumbran un mejor panorama en el ejercicio de sus funciones académicas, a fin de que estas coadyuven al desarrollo y rendimiento académico en cuanto a los saberes, conocimientos, métodos y técnicas, que son indispensables para los logros de los fines educativos.

REVISTA ANUAL ACCIÓN Y REFLEXIÓN EDUCATIVA, N 45

enero, 2020

ISSN L 2664-3775 
En consecuencia, a lo arriba aludido, es importante el conocimiento del modelo de gestión a utilizar, como sustento teórico en su aplicación sobre los hechos empíricos en la institucionalidad educativa, puesto que en tales procesos el mismo está acompasado con el desempeño de los actores, para lograr los objetivos y metas propuestos, a corto y largo plazo en el Centro Educativo.

Ante esta situación surgieron interrogantes, que se utilizaron para realizar un análisis a priori sobre la base de algunas teorías de gestión. En esta oportunidad serían los actores educativos, los responsables de evaluar, la eficacia o no del modelo de gestión adoptado por los centros escolares, a partir de la aprobación del Decreto 155 del 27 de marzo de 2014.

Por lo que se presentaron las siguientes interrogantes:

¿Qué sucede cuando la gestión educativa, no rinde los resultados esperados, y el director de escuela no se percata? ¿Cuál es la posibilidad de éxito de una gestión educativa, sin participación de los actores sociales involucrados? ¿Existe la posibilidad de mejorar la gestión de los centros, con la adopción de un modelo definido por todos?

En la búsqueda de respuestas se encontró que, en el territorio panameño, la Ley 47 Orgánica de Educación, establece que en:

Cada centro educativo estará a cargo de un Director o Directora, que será jefe o superior inmediato del personal docente y administrativo que labora en el plantel $y$, por tanto, el funcionario responsable ante el Ministerio de Educación, de la buena marcha de la institución que dirige.

REVISTA ANUAL ACCIÓN Y REFLEXIÓN EDUCATIVA, $\mathrm{N}^{\circ} 45$

enero, 2020

ISSN L 2664-3775 
En este sentido, es responsable de todos los procesos de gestión educativa, asumiendo el compromiso de lograr las mejores condiciones posibles para el cumplimiento de los fines educativos, en todos los niveles que regente el centro.

Es urgente y necesario que, desde la planificación estratégica, se logre que los actores sociales (autoridades ministeriales y escolares in situ, docentes en servicio, supervisores regionales según zonas escolares, y las colaboraciones y compromisos y cumplimientos académicos, sociales y culturales por parte de los estudiantes), en cuanto al alcance de una gestión exitosa deberá incluir aspectos fundamentales, tales como: programación, organización, dirección, ejecución y el control. Esto demanda del director de escuela poseer las competencias, para actuar en todos los ámbitos; y lo obliga a fortalecer continuamente sus conocimientos, habilidades, capacidades y destrezas pedagógicas y administrativas, para ejercer apropiadamente la función directiva.

Sin embargo, en la Región Educativa de Panamá Centro, como en el resto de las regiones educativas del país, se percibe la práctica de una cultura del empirismo, por la falta de formación para el ejercicio de la función directiva, probablemente como resultado de la inexistencia de políticas, programas, proyectos y acciones, resultantes de diagnósticos con rigor científico. Al respecto se puede decir que esto puede resultar peligroso; ya que los directores de centros educativos son los responsables de garantizar que el uso de los recursos asignados, coadyuven al logro de los objetivos y alcance de las metas propuestas.

No obstante, la normativa de la educación panameña establece que: En cada centro escolar del primer y segundo nivel de enseñanza, funcionará un organismo, consultivo y de participación ad honórem, denominado Comunidad Educativa Escolar... (Ley 47 de 1946, Orgánica de Educación, Art.51). Este organismo está comprometido en cumplir con la responsabilidad Constitucional, de colaborar en la gestión total del centro y acompañar al director en el diseño ejecución y control de los planes y proyectos que se desarrollen.

REVISTA ANUAL ACCIÓN Y REFLEXIÓN EDUCATIVA, N 45

enero, 2020

ISSN L 2664-3775 


\section{- Plan de mejora al contexto de los centros educativos panameños}

Para mejorar los resultados, es obligante pensar en un medio que permita a los actores sociales, reconocerse como tales, para determinar el estado de situación del centro escolar en términos sociales, pedagógicos, administrativos y de infraestructura, para luego proyectar un punto a alcanzar y trazarse un camino compartido por todos.

El Ministerio de Educación de Panamá, a través del Decreto Ejecutivo 155 del 27 de marzo de 2014; dejó sentada las bases para la elaboración del Plan de Mejora Escolar, en cuanto a que "está "dirigido a promover y garantizar la calidad de la educación y de los demás servicios que ejecutan las instituciones educativas oficiales de primer y segundo nivel de enseñanza del país". Esta iniciativa se fundamenta en la Ley 47 Orgánica de Educación de 1946, que estipula:

La evaluación educativa del sistema se realizará de acuerdo a principios que la hagan científica, integral, continua, acumulativa y participativa... dicha labor abarcará elementos de evaluación institucional y de los aprendizajes, a fin de garantizar la eficiencia y eficacia de funcionamiento del sistema. (artículo 343).

\section{Evaluación institucional}

Los procesos del SECE, se apoyan en los logros obtenidos por los centros educativos como organización o institución; centrados en la autoevaluación de evidencias de la gestión administrativa, pedagógica y trabajo conjunto con las familias y la comunidad. Estos procesos atienden la participación de los agentes implicados en la educación (directivos, docentes, administrativos, familia y estudiantes), el compromiso de los funcionarios nombrados en el centro educativo, la colaboración de la comunidad escolar, el trabajo en equipo y el mejoramiento de los servicios y del recurso humano de la organización, así como es la participación por parte de todos los actores.

REVISTA ANUAL ACCIÓN Y REFLEXIÓN EDUCATIVA, N 45

enero, 2020

ISSN L 2664-3775 


\section{- Participación de los actores socioeducativos}

Los actores socioeducativos son todos aquellos entes sociales que de una u otra forma se ven involucrados en los planes, proyectos, actividades o acciones que se realizan en el centro educativo y su entorno circundante.

La importancia que adquiere la participación en el entorno de la educación, hace necesario definir el concepto:

La participación puede entenderse como aquello a lo que pertenecemos como seres biológicos, sociales, políticos y que nos influye (las condiciones base de nuestra existencia) o aquello otro que asumimos con capacidad volitiva, responsabilidad y libertad (las construcciones de nuestra existencia), en lo que decidimos tomar parte (García R., 2004, p.65).

Velasco (2000) citado por Obando, (2008), asegura que "la participación es la intervención de personas o grupos en la discusión y toma de decisiones, que los afectan para el logro de determinados objetivos" (p. 91). En otras palabras, debe ser el medio que contribuya a unificar criterios entre los actores socioeducativos; además de sensibilizar para enfrentar el cambio.

El concepto de participación se utiliza con mucha frecuencia en el campo de la planificación educativa, para referirse a la necesidad de los beneficiarios de las políticas, programas y proyectos de involucrarse activamente en el logro de las metas y objetivos.

Los actores socioeducativos de los centros escolares son los responsables directos de ejecutar los procesos del SECE, los cuales en primera instancia serían: 1. Autoevaluación; 2. Formulación del plan de mejora; 3. Aplicación del plan de mejora.

REVISTA ANUAL ACCIÓN Y REFLEXIÓN EDUCATIVA, N 45

enero, 2020

ISSN L 2664-3775 
De acuerdo al Decreto 155 de 27 de marzo de 2014:

En cada centro educativo funcionará una Comisión Institucional de Evaluación del Centro Educativo, que se encargará de la organización, ejecución y seguimiento de los procesos del SECE en la institución de enseñanza; esta comisión tiene la representatividad de cada estamento que conforma la comunidad educativa, para cumplir con cada uno de los procesos (artículo 15).

\section{- Los procesos del SECE}

Los procesos del sistema están conjugados con elementos que contienen para sus eficiencias y desarrollos los aspectos endógenos de la autoevaluación; paralelo a ello, se aplica un plan de mejoras, con el propósito de alcanzar que los procesos del SECE, sean efectivos, dinámicos eficientes y con calidad de gestión.

\section{- Autoevaluación}

Se trata de realizar un análisis interno, para dar cuenta de lo que se ha venido haciendo como centro educativo. No es individualizada, se busca que todos los actores de un centro educativo realicen una mirada introspectiva que les permita ver cómo cada uno ha venido cumpliendo sus deberes y cuán eficaces han sido. A partir de esta autoevaluación viene el siguiente paso.

\section{- Plan de mejoras}

Es el proceso que asegura que cada centro educativo esté comprometido con mejorar sus debilidades, consolidar sus fortalezas en un proyecto en el que todos conocen el punto de partida, el camino a recorrer y la meta que, como equipo, deciden aspirar. O sea, cada centro educativo construye su Plan de Mejora, usando como insumo los resultados del proceso de Autoevaluación.

REVISTA ANUAL ACCIÓN Y REFLEXIÓN EDUCATIVA, N 45

enero, 2020

ISSN L 2664-3775 


\section{- Aplicación del Plan de Mejora}

A partir de lo planificado, el siguiente paso consiste fundamentalmente en acompañar y dar seguimiento a la puesta en marcha del plan, con miras a que al final, se validen los avances y logros de las acciones; y que con posterioridad la propia comunidad educativa proyecte los planes siguientes.

\section{MATERIAL Y MÉTODOS}

En esta investigación se esbozó un diagnóstico que apuntó por describir todo el proceso que relaciona la participación cooperativa y colaborativa por parte de los docentes hacia los estudiantes y padres de familias, y viceversa, los padres de familia, los estudiantes y docentes; generando una sinergia activa que ayuda al desarrollo neuro-cognitivo y las prácticas intra-muros y extra- muros, repotenciándose lo académico con relación al rendimiento, éxitos escolares y la participación de la comunidad en la que se encuentra el CEBG, dándole fortaleza y abriendo oportunidades para concretar un plan de mejoras a los respectivos centros y actores sociales, objetos de investigación.

En este aspecto metodológico, la investigación se justificó sobre la base de la necesidad de indagar el desarrollo en las formas de gestión que se evidencian en las concreciones a través de las argumentaciones que fueron identificadas y señaladas, las causalidades aludidas, se pudieron observar las delineaciones cuyas formas son estructuralmente rígidas (infraestructura, equipamiento técnico, mobiliario, etc.) y, por la otra parte, las relacionadas con la participación de los actores sociales (docentes-estudiantes-padres de familias-autoridades ministeriales educativas) las que involucraron en estas gestiones de acciones, el desarrollo de comportamientos que aunaron a que los sentimientos, estados motivacionales, compromisos y responsabilidades, se convirtieron en elementos reeponteciadores y competenciales sobre el 
proceso de planificación de las escuelas, y de manera muy particular, sobre el diseño e implementación del Plan de Mejora Escolar en el CEBG Ciudad Jardín las Mañanitas, CEBG Reino de los Países Bajos y CEBG Cirilo J. Martínez, de la Región Escolar de Panamá Centro, de la Provincia de Panamá, ocurriendo en estos centros escolares una optimización de los mismos, identificándose y señalándose lineamientos que fueron aflorados en el diseño y ejecución del proceso de autoevaluación, y con ello, las propuestas de mejoras de los resultados de la Gestión del Centro Escolar; lográndose como finalidad última, el continuar con una gestión dinámica, motivadora, alicientes para los actores sociales involucrados en las instituciones pioneras en este modelo administrativo.

El enfoque metodológico utilizado fue de carácter no-experimental, de tipo exploratorio. Para el desarrollo de la investigación se utilizaron como apoyo libros especializados en el tema, investigaciones realizadas en este campo, documentos de Internet, encuestas y entrevistas, entre otras fuentes. Para darle fundamentación técnica e instrumental, la misma se amparó en los teóricos mexicanos Hernández; Fernández; y Baptista (2010, p. 149), por ello, "se utilizó una muestra, no probabilística intencional de sujetos"; se aplicó la técnica de la encuesta y la entrevista abierta en cuanto a la recolección y registro, que previamente fueron seleccionado por la muestra de los informantes: directivos administrativos, docentes y los miembros de las directivas de las asociaciones de padres de familia.

En base a los criterios muéstrales por conveniencia (no paramétricos), se consideró trabajar con una muestra del 30\% del total de los docentes de cada escuela, el $100 \%$ del total de directivos administrativos y el $100 \%$ total de los miembros directivos de las asociaciones de padres de familia. 
Quedando representada con un universo poblacional y muestra por conveniencia, y distribuida así:

- De 205 docentes de las tres escuelas sedes del estudio. Se trabajó con una muestra de sesenta y dos (62) docentes,

- El total de treinta (30) miembros Juntas directivas de las asociaciones,

- Y ocho (8) Directivos administrativos de las tres escuelas sedes.

Se articuló un marco teórico sobre el cual se identificaron y seleccionaron, aspectos o situaciones relacionadas a los actores sociales participantes y sus aspiraciones reales para mejorar la calidad y los servicios ofrecidos y recibidos en el proceso de gestión que se desarrollan en los CBEG, pero también aquellos aspectos materiales constructivos, mantenimientos de la edificaciones, y embellecimiento de ornatos y limpiezas, en los que se evidenciaron las utilidades para que la comunidad educativa escolar, regional y nacional, encausen soluciones pertinentes a problemas comunes de participación comunitaria y de gestión en el contexto pedagógico, administrativo y social; dentro y fuera de las instituciones educativas de todo el país.

En este sentido, se conoció y definieron teorías que ayudaron a encausar desde el análisis, los niveles de participación de la Comunidad Educativa Escolar, y su aporte al diseño y ejecución del Plan de Mejora en (3) tres escuelas de la Región Escolar de Panamá Centro. Esto como base para intentar describir la relación de los docentes con los padres de familia y otros miembros de la comunidad educativa escolar. Así como identificar qué motivaciones tienen los diversos actores sociales para participar en la organización integral del centro; entre tantos factores que viabilizan o dificultan la participación de los docentes, padres de familia y otros miembros de la comunidad educativa escolar en la planificación educativa del centro.

REVISTA ANUAL ACCIÓN Y REFLEXIÓN EDUCATIVA, N 45

enero, 2020

ISSN L 2664-3775 
La sistematización del proceso general de la implementación del proyecto de investigación se realiza confrontado con su diseño, permitiendo disponer de una alternativa para la planeación normativa que puede ser transferible a diversos centros escolares o para instituciones de otra índole, dado que se puede contar con un modelo y un material accesible, que sólo precisa ser mejorado o reconstruido de acuerdo con la realidad de cada organización.

La hipótesis teórica, pudo contrastarse de acuerdo a los segmentos teóricos esbozados, y que luego, a través del análisis causativo del proceso de planificación que ocurren en las escuelas antes mencionadas, se tomaron las valoraciones individualizadas que sienten y desean los actores sociales, de esta realidad fáctica o real, es concluyente ante la necesidad de diseñar un Plan de Mejora Escolar, para el logro de resultados óptimos en los centros que regenta la región educativa, por ello la hipótesis planteada, tomó la base material fáctica y el deseo actitudinal y comportamental en los actores sociales participantes, "La participación real y efectiva, de la Comunidad Educativa Escolar, es la mejor posibilidad, para el diseño y ejecución del Plan de Mejora en las escuelas de la Región Escolar de Panamá Centro". Lo cual se traduce en beneficios expresados en el mejoramiento continuo de los procesos de planificación, diseño, monitoreo, evaluación seguimiento y evaluación de los procedimientos aplicados, a fin de mejorar la calidad, el control de la gestión, la satisfacción y la respuesta de toda la comunidad educativa además de mantener un nivel de bienestar y equilibrio interno.

El diseño de investigación propuesto, está apegado al método cualitativo, es decir, en cuanto a que hay descriptores que permiten las identificaciones categoriales, conceptuales y teóricas, que se explicaron operativamente bajo parámetros del orden cuantitativo. Esta se amparó al acumulado teórico esbozado por las contrastaciones empíricas comprobatorias por intermedio del cálculo y tamaño de la muestra por conveniencia, haciendo praxeológicamente que esta perspectiva cualitativa sea pertinente según el empleo de este paradigma. Por esa razón teórica (cualitativa) y técnica (cuantitativa), es que:

REVISTA ANUAL ACCIÓN Y REFLEXIÓN EDUCATIVA, $\mathrm{N}^{\circ} 45$

enero, 2020

ISSN L 2664-3775 
La investigación cualitativa trata de identificar la naturaleza profunda de las realidades, su sistema de relaciones, su estructura dinámica. La investigación cuantitativa trata de determinar la fuerza de asociación o correlación entre variables, la generalización y objetivación de los resultados a través de una muestra para hacer inferencia a una población de la cual toda muestra procede (Guerrero Dávila \& Guerrero Dávila, 2000, p. 47).

La sistematización del proceso general de la implementación del proyecto de investigación se realiza confrontado con su diseño, permitiendo disponer de una alternativa para la planeación normativa que puede ser transferible a diversos centros escolares o para instituciones de otra índole, dado que se puede contar con un modelo y un material accesible, que sólo precisa ser mejorado o reconstruido de acuerdo con la realidad de cada organización.

\section{ANÁLISIS DE RESULTADOS}

Los docentes encuestados representan el 30\% del total que laboran en las tres escuelas sedes del estudio. Por su parte, los directivos y personal administrativo encuestados representan el 88\% del total. En relación a los miembros de juntas directivas de las asociaciones de las respectivas escuelas, representan el 100\%.

A pesar de los años que lleva la implementación de la Ley 50, se dan resultados interesantes, por ejemplo en la pregunta ¿Conoce usted el término Comunidad Educativa (CE)?, se puedo constatar que el $84 \%$ de los docentes contestaron que totalmente conocían el término, mientras que el 16\% afirmó que parcialmente; dentro del estamento padres de familia el 17\% aseveró no conocer nada referido al término y el $83 \%$ aseguró conocer el término, parcial o totalmente; y como era de esperarse el 100\% de los directivos certificó conocerlo, totalmente. Al realizar un cruce entre los resultados con el ítem de formación o grado académico, encontramos que los padres de familia que aseguraron conocer "nada" del término, poseen grado académico 
intermedio, o sea Educación Media. Por lo que pareciera, no existir relación entre los niveles de estudio y el conocimiento del término "Comunidad Educativa".

Al preguntar si se sentían parte de la Comunidad Educativa (CE) el 13\% de los padres de familia afirmó no se sentirse parte; mientras que el $47 \%$ de los padres se siente parte, parcialmente; al igual que el $21 \%$ de los docentes. El resto de los actores afirmó sentirse totalmente parte de la CE. Mostrando un nivel de pertenencia aceptable en las tres escuelas sedes del estudio.

En cuanto a la interrogante ¿Participa usted de las acciones que realiza la Comunidad Educativa (CE)?, el 100\% de los directivos asegura participar de todas las acciones que se realizan en la escuela, respuesta muy coherente con la responsabilidad del puesto que ostentan; sin embargo el $29 \%$ de los docentes afirmó participar parcialmente o en nada que organice la escuela y el $71 \%$ indicó que participan en todo lo que se realice; por su parte los padres de familia evidencian una relación directa con la respuesta del ítem anterior, ya que los mismos individuos que representan el 13\% aseveraron no participar de las acciones que realiza el CE y el $87 \%$ dijo participar total o parcialmente.

Los resultados de este ítem muestran un nivel de participación medianamente alto por parte de los docentes en las acciones que realiza el CE; contrario a los niveles altos de participación que reflejan directivos y padres de familia. Lo que refleja las relaciones propias de la escuela con su entorno.

Hablar de las relaciones de un centro con su entorno interpela a su propia identidad como organización. Si un centro no tiene entidad ni identidad propia difícilmente podrá establecer relaciones con su entorno de forma sistemática y coherente con sus fines. Habrá, a lo sumo, intervenciones puntuales, individuales, paralelas, al margen, extraordinarias, complementarias, ocasionales,... No habrá 
relaciones de CENTRO con la COMUNIDAD, sino de un profesor, un grupo, una asignatura, unos padres... (San Fabián, 1996, p.196).

Cada día, cobra vigencia la integración de los agentes socioeducativos a las actividades de la escuela y éstas son vistas como un elemento fundamental para coadyuvar a la obtención de mejores resultados en la gestión educativa.

Tanto así que el plan de mejora es conocido por todos los actores socioeducativos encuestados, en los centros educativos que fueron sedes del estudio. Cuarenta y seis (46) docentes y dieciocho (18) padres de familia contestaron que conocen totalmente el plan de mejora escolar, al mismo tiempo catorce (14) docentes y once (11) padres de familia dijeron conocer parcialmente el plan de mejora. Solo dos (2) docentes y un (1) padre de familia dijeron no conocer nada del plan de mejora escolar.

La participación de padres y docentes está por encima del 76\% en ambos casos. Indicativo que el proceso de autoevaluación y elaboración del plan de mejoras, en los centros educativos sede del estudio, se cumplió con uno de los objetivos del SECE, que a su letra dice “...procurar el mejoramiento de la gestión institucional que favorezca la comunicación entre los miembros..." (Decreto Ejecutivo 155, 2014)

Se observó también, que la mayoría de los padres y docentes encuestados, creen que las metas propuestas en el plan de mejora se alcanzaron parcial o totalmente, coincidentemente los padres $\mathrm{y}$ docentes que afirmaron no creer que se alcancen las metas; tampoco participaron en la elaboración del plan de mejoras.

Es crítico para el éxito de un proyecto identificar los actores interesados (stakeholder), tan pronto como sea posible, desde el comienzo de su ciclo de vida. "El director del proyecto decide qué nivel de participación y compromiso con el proyecto debe tener cada stakeholder" (Project Management Institute, 2008. p.441). Se logra constatar los altos niveles de pertenencia por parte

REVISTA ANUAL ACCIÓN Y REFLEXIÓN EDUCATIVA, N 45

enero, 2020

ISSN L 2664-3775 
de los actores socioeducativos (stakeholder), debido a que más del 93\% de los encuestados asegura que el plan de mejora, fue consultado a la comunidad educativa (CE), parcial o totalmente.

Respecto a la interrogante: "existe comunicación entre los docentes y padres de familia", encontramos que el $81 \%$ de los docentes dijo que existe total comunicación y un 19\% afirmó que la comunicación es parcial. Mientras que a la misma pregunta el 63\% de los padres de familia, dijo que la comunicación es total y el 37\% afirma que se comunican parcialmente.

Si la comisión de evaluación institucional o escolar reconoce, la influencia que puede tener una efectiva estrategia de comunicación; además de lograr que se cumplan las metas, ésta puede contribuir a formar lazos y/o alianzas estratégicas, para acrecentar la productibilidad de la organización.

Ante la pregunta: ¿El Plan de Mejora contribuye a mejorar los resultados de la Gestión del Centro?; el estudio reveló que el $85 \%$ de los docentes se inclina por decir que está totalmente convencido de su contribución; mientras que el 11\% asegura que parcialmente contribuirá a mejorar los resultados del centro. Por su parte el 90\% de los padres de familia aseguró que totalmente y un $10 \%$ dijo que parcialmente. Y solo el $3 \%$ de los docentes señaló que no mejorará en nada.

\section{CONCLUSIONES}

Los niveles de participación de la Comunidad Educativa Escolar, en las escuelas CEBG Ciudad Jardín las Mañanitas, CEBG Reino de los Países Bajos y CEBG Cirilo J. Martínez, de la Región Escolar de Panamá Centro, de la Provincia de Panamá, son altos y esta situación viabiliza el

REVISTA ANUAL ACCIÓN Y REFLEXIÓN EDUCATIVA, N 45

enero, 2020

ISSN L 2664-3775 
aporte de éstos al diseño y ejecución del plan de mejora y a otras acciones que se realicen en los centros escolares.

La relación y efectividad de la comunicación entre los actores socioeducativos, se convirtió en la clave que contribuyó a detectar problemas, para lograr un mejor desempeño en los procesos internos e incrementar la productividad colaborativa como resultado del trabajo participativo.

Entre los factores que dificultan la participación de los docentes, padres de familia y otros miembros de la comunidad educativa escolar en la planificación educativa del centro, se mencionan con mayor incidencia la falta de colaboración y la burocracia estatal.

Los padres de familia y otros miembros de la comunidad educativa escolar demandan, frente a la realidad educativa, la creación de más escenarios de participación, para la toma de decisiones en todos los niveles del sistema educativo.

Los factores asociados a la participación de los docentes, padres de familia y otros miembros de la comunidad educativa escolar en la planificación educativa del centro, no son de carácter instrumental y de control absoluto del administrador del proyecto.

El grado académico, de los actores socioeducativos no influye en los niveles de participación, contrario a lo que han señalado otros autores quienes aseguran que un bajo nivel de conocimiento de los procesos de planificación y participación puede ser un factor asociado, que incide en la disposición a participar de los miembros de la comunidad educativa.

Se debe considerar la participación real y efectiva, de la Comunidad Educativa Escolar, "como un factor de éxito", para el diseño y ejecución del Plan de Mejora en las escuelas de la Región Escolar de Panamá Centro; así como en otras acciones que demande el sistema educativo, para mejorar los resultados obtenidos.

REVISTA ANUAL ACCIÓN Y REFLEXIÓN EDUCATIVA, N 45

enero, 2020

ISSN L 2664-3775 
La lección aprendida de este proceso, y que consideramos de suma importancia, es que es posible cambiar los resultados en educación y que uno de los caminos para lograrlo es transformando la gestión de los centros educativos. Una transformación que lo atienda holísticamente y tome en cuenta la dimensión pedagógica, social, administrativa y de infraestructura, pero que además se ajuste al contexto geográfico, cultural, económico y social del propio centro escolar. Y es que un centro educativo, cambiará realmente si cambia su cultura organizacional.

Sin duda el ejercicio de autoevaluación y elaboración del Plan de Mejora escolar han significado un avance en los niveles de participación al interior de estos establecimientos escolares, si tomamos en cuenta que éstos históricamente han sido espacios de autoritarismo y territorios exclusivos del "claustro académico". Esta iniciativa permite que todos los estamentos se reúnan, dialoguen y compartan sus expectativas con los otros, y a su vez aumenta el sentido de pertenencia a la comunidad educativa del centro. No obstante, se requiere del desarrollo de una política oficial que fortalezca la participación, la descentralización y desconcentración en la toma de decisiones.

REVISTA ANUAL ACCIÓN Y REFLEXIÓN EDUCATIVA, N 45 enero, 2020 


\section{REFERENCIAS}

Alarcón, M. (2008). Planificación Estratégica: Una Herramienta en la gestión escolar a nivel de educación Básica. Recuperado de http://cidar.uneg.edu.ve/DB/bcuneg/EDOCS/TESIS/TESIS_POSTGRADO/MAESTRIAS/CI ENCIAS EDUCACION/TGMLA53T462008.pdf/

Antúnez, S. (2012). Una brújula para la dirección escolar. Orientaciones para la mejora. México: Editorial Somos Maestros-Innovación y Asesoría Educativa. Serie Gestión Educativa.

Gaceta Oficial. (2004). Decreto Ejecutivo 305 del 30 de abril de 2004, aprueba el Texto Único de la Ley 47 de 1946, Orgánica de Educación. Gaceta Oficial Digital, № 25042.

Gaceta Oficial. (2014). Decreto Ejecutivo 155 del 27 de marzo de 2014, que crea el sistema de evaluación en los centros educativos. Gaceta Oficial Digital, No 27502.

García, R. (2004). Políticas y programas de participación social. Madrid: Editorial Síntesis.

Guerrero Dávila, G., y Guerrero Dávila, M. (2000). Metodología de la Investigación, Serie Integral por Competencias DGB. México: Grupo Editorial Patria.

Harf, R. and Azzerboni, D. (2009). Estrategias para la acción directiva. Madrid: Humanes de Madrid, CEP.

Hernández, R. y Otros. (2010). Metodología de la investigación. México: Editorial Mc Graw Hill.

Ministerio de Educación. (2014). Plan de Mejoras - Guía Práctica de Implementación. Panamá: Imprenta Educativa - MEDUCA.

REVISTA ANUAL ACCIÓN Y REFLEXIÓN EDUCATIVA, N 45

enero, 2020

ISSN L 2664-3775 
Obando Castillo, G. (2008). "La participación docente en la toma de decisiones desde la visión micropolítica”. Revista de Educación ( $\left.{ }^{\circ} 32\right)$, pp.87-108.

Project Management Institute (PMI). (2008). Guía de los Fundamentos para la Dirección de Proyectos (Guía del PMBOK). 4ª Edición. Project Management Institute.

Salinas C., M. C. (2003). "Planeación normativa como base para generar un proceso de planeación institucional con enfoque participativo”. Transferencia-Revista Digital de Posgrado, Investigación y Extensión del Campus de Monterrey. Año 17 - Número 66 - Abril de 2004. Recuperado de http://www.mty.itesm.mx/die/ddre/transferencia/66/66-II.05.html.

San Fabián Maroto, J. (1996). "El centro escolar y la comunidad educativa: ¿un juego de metáforas?". Revista de Educación (N. 309), pp. 195-215.

Sánchez Fernández, M. (2014). Comunicación efectiva y trabajo en equipo. Madrid: Humanes de Madrid, CEP. 